\title{
Research Article \\ Shear Stress Counteracts Endothelial CX3CL1 Induction and Monocytic Cell Adhesion
}

\author{
Aaron Babendreyer, Lisa Molls, Daniela Dreymueller, Stefan Uhlig, and Andreas Ludwig
}

Institute of Pharmacology and Toxicology, RWTH Aachen University, Aachen, Germany

Correspondence should be addressed to Andreas Ludwig; aludwig@ukaachen.de

Received 13 January 2017; Accepted 23 February 2017; Published 26 March 2017

Academic Editor: Magdalena Riedl

Copyright (c) 2017 Aaron Babendreyer et al. This is an open access article distributed under the Creative Commons Attribution License, which permits unrestricted use, distribution, and reproduction in any medium, provided the original work is properly cited.

\begin{abstract}
Flow conditions critically regulate endothelial cell functions in the vasculature. Reduced shear stress resulting from disturbed blood flow can drive the development of vascular inflammatory lesions. On endothelial cells, the transmembrane chemokine CX3CL1/ fractalkine promotes vascular inflammation by functioning as a surface-expressed adhesion molecule and by becoming released as soluble chemoattractant for monocytic cells expressing the receptor CX3CR1. Here, we report that endothelial cells from human artery, vein, or microvasculature constitutively express CX3CL1 when cultured under static conditions. Stimulation with $\mathrm{TNF} \alpha$ under static or very low shear stress conditions strongly upregulates CX3CL1 expression. By contrast, CX3CL1 induction is profoundly reduced when cells are exposed to higher shear stress. When endothelial cells were grown and subsequently stimulated with TNF $\alpha$ under low shear stress, strong adhesion of monocytic THP-1 cells to endothelial cells was observed. This adhesion was in part mediated by transmembrane CX3CL1 as demonstrated with a neutralizing antibody. By contrast, no CX3CL1-dependent adhesion to stimulated endothelium was observed at high shear stress. Thus, during early stages of vascular inflammation, low shear stress typically seen at atherosclerosis-prone regions promotes the induction of endothelial CX3CL1 and monocytic cell recruitment, whereas physiological shear stress counteracts this inflammatory activation of endothelial cells.
\end{abstract}

\section{Introduction}

In the body, vascular endothelial cells are constantly exposed to blood flow. The resulting laminar shear stress on the endothelial surface can largely differ in large and small arteries or veins, respectively $[1,2]$. Exposure to laminar flow typically leads to an alignment of the endothelial cells in the direction of the flow, cytoskeletal rearrangements, the formation of tight endothelial cell-to-cell contacts, reduced permeability, arrest of cell proliferation, and prolonged cell survival [3-7]. These phenotypical characteristics are maintained by a transcriptional response to the flow conditions resulting in the altered expression of regulators of the vascular tone, endothelial surface molecules, and soluble mediators. Typically, endothelial NO synthase is upregulated by high shear stress, whereas endothelin-1 or vascular cell adhesion molecule-1 (VCAM-1) gene expression is downregulated [8-12].

The importance of shear stress for endothelial cell functions is highlighted by pathological processes associated with reduced or absent laminar shear stress, which can occur in vascular beds that are prone to atherosclerosis. At branch points and curved areas such as the carotid sinus, disturbed flow including flow reversal or turbulence may occur. In these areas, the average shear stress is considerably lower and values below $1 \mathrm{dyn} / \mathrm{cm}^{2}$ can occur, which is 10 times lower than the average of $10 \mathrm{dyn} / \mathrm{cm}^{2}$ in the human vasculature and even 100 times lower compared to the microvasculature $[1,2,13]$. These areas are characterized by an absence of preferential endothelial alignment and are predisposed to develop atherosclerotic lesions. An early step in the development of atherosclerotic lesions is the production of proinflammatory chemokines and adhesion molecules by the vascular wall. This results in increased binding of inflammatory leukocytes, especially monocytes, from the blood to the vascular surface. The adherent monocytes become activated and migrate into the vascular wall where they contribute to the lesion development by production of inflammatory mediators, by uptake of lipids, and by differentiation into macrophages and finally foam cells [14]. 
Monocyte recruitment to atherosclerotic lesions is driven by several chemotactic cytokines including CX3CL1 also termed fractalkine [15]. CX3CL1 is special within the chemokine family, since it is synthesized as a transmembrane molecule that is expressed on the endothelial cell surface [16]. As such, the chemokine can interact with its receptor CX3CR1, which is expressed on monocytic cells, T cell subsets, and NK cells [17]. Thereby, CX3CL1 can promote cell adhesion without the need of further activation [18]. Stimulation of cultured endothelial cells with proinflammatory mediators leads to enhanced expression of CX3CL1, which then promotes adhesion of monocytic cells. Thereafter, the activation of CX3CR1 can induce cell migration of the adherent monocytes leading to transmigration through the endothelial cell layer [19]. In addition to its activity as transmembrane adhesion molecule, CX3CL1 can act as classical chemoattractant for leukocytes when it is shed from the cell surface by the activity of ADAM10 (short for a disintegrin and metalloproteinase) and ADAM17 [20, 21]. Besides CX3CL1, the only other transmembrane chemokine is CXCL16, which is also expressed and shed by endothelial cells $[22,23]$ and implicated in the recruitment of $\mathrm{T}$ cells to the inflamed vasculature $[24,25]$.

It has been shown that CX3CL1 is highly expressed at vascular sites, which are prone to lesion development and serve as one of the critical mediators driving monocytic cell recruitment to these lesions [26, 27]. In carotid arteries, CX3CL1 expression is highest at regions of low laminar shear stress [28]. This may suggest that the upregulation of the chemokine can be a direct result of the altered flow conditions in these regions. In the present report, we further address this question by performing flow experiments with cultured endothelial cells. We show that endothelial cells of different vascular beds respond to flow conditions by downregulating the gene expression of CX3CL1. Moreover, flow conditions suppress the induction of CX3CL1 in response to $\mathrm{TNF} \alpha$. We then show that the reduced surface expression of CX3CL1 on endothelial cells results in reduced adhesion of monocytic cells. Thus, physiological shear stress acts as atheroprotection by downregulating CX3CL1 expression to maintain a noninflammatory phenotype of endothelial cells.

\section{Material and Methods}

2.1. Antibodies, Chemokines, and Inhibitors. Biotinylated mouse monoclonal antibody against human CX3CL1 (clone 51637), mouse monoclonal antibody against human CX3CL1 (clone 51637 and clone 81506), goat polyclonal antibody against human CXCL16, and mouse IgG1 isotype control were from R\&D Systems (Wiesbaden, Germany). Biotinylated rabbit polyclonal antibody against human CXCL16, human CX3CL1, human CXCL16, and human TNF $\alpha$ were from PeproTech (Rocky Hill, USA). Allophycocyanin- (APC-) conjugated goat antimouse antibody was from Jackson ImmunoResearch Laboratories, Inc. (West Grove, USA). Calcein-AM was from BIOTREND Chemikalien $\mathrm{GmbH}$ (Cologne, Germany).
2.2. Cell Culture. Monocytic THP-1 cells were cultured in RPMI (Sigma-Aldrich, St. Louis, USA) with 10\% FBS (PAN-Biotech, Aidenbach, Germany) with a cell density of 0.2 to $2.0 \times 10^{6}$ cells per $\mathrm{ml}$.

Human pulmonary microvascular endothelial cells (HPMECs) were from PromoCell (Heidelberg, Germany) and cultured in Endothelial Cell Growth Medium MV2 (PromoCell) as recommended by the supplier. Human umbilical vein endothelial cells (HUVECs) and human umbilical artery endothelial cells (HUAECs) were isolated from the umbilical cord of caesarean sections in our laboratory as described [19] and cultured in Endopan-3 from PANBiotech (Aidenbach, Germany).

For flow experiments, cells were used in passages 4 to 6 and seeded with a density of 100,000 cells $/ \mathrm{cm}^{2}$ in ibidi $\mu$-Slides of different types $(0.1,0.2,0.4$, and $0.8 \mathrm{~mm} \mu$-Slides, Martinsried, Germany) or 24 well plates for the static control. After $24 \mathrm{~h}$, supernatants and cells were harvested or stimulated with $10 \mathrm{ng} / \mathrm{ml} \mathrm{TNF} \alpha$ for another $24 \mathrm{~h}$. The flow in the $\mu$-Slides was created with the ibidi pump system. Depending on the geometry of the $\mu$-Slide type, the flow rate was adjusted as specified by the manufacturer to yield the desired laminar shear stress.

2.3. $R T-q P C R$. The mRNA levels for CX3CL1 and CXCL16 were quantified by RT-qPCR analysis and normalized to the mRNA level of GAPDH (glyceraldehyde 3-phosphate dehydrogenase). For the first set of flow experiments, a set of housekeeping genes (GAPDH, TATA-binding protein-TBP, and cytochrome $\mathrm{C} 1-\mathrm{CYC} 1)$ were used as reference genes instead of only GAPDH. RNA was extracted using RNeasy Kit (Qiagen, Hilden, Germany) and quantified using NanoDrop (Peqlab, Erlangen, Germany). Equal amounts of mRNA within each data set were reversely transcribed using PrimeScript $^{\mathrm{TM}}$ RT Reagent Kit (Takara Bio Europe, St-Germain-enLaye, France) according to the manufacturer's protocol. PCR reactions were performed using SYBR Premix Ex Taq II (Takara Bio Europe) according to the manufacturer's protocol. The following primers were used with the specific primer annealing time given in brackets: CX3CL1 (forward: GGTTAGGCATTGTGGGAAGG, reverse: AGATGGGAG TATGTTGGTGG) $\left(60^{\circ} \mathrm{C}\right)$, CXCL16 (forward: TGTCTATA CTACACGAGGTTCCA, reverse: AGCATGTCCACATTC TTTGAG) $\left(60^{\circ} \mathrm{C}\right), \mathrm{GAPDH}$ (forward: CGGGGCTCTCCA GAACATCATCC, reverse: CCAGCCCCAGCGTCAAAG GTG) $\left(66^{\circ} \mathrm{C}\right), \quad T B P$ (forward: GAGCCAAGAGTGAAGA ACAGTC, reverse: GCTCCCCACCATATTCTGAATCT) $\left(60^{\circ} \mathrm{C}\right)$, and $\mathrm{CYC1}$ (forward: AGCTATCCGTGGTCTCA CC, reverse: CCGCATGAACATCTCCCCATC) $\left(59^{\circ} \mathrm{C}\right)$. All PCR reactions were run on a LightCycler ${ }^{\circledR} 480$ System (Roche, Basel, Switzerland) with the following protocol: 45 cycles of $10 \mathrm{~s}$ denaturation at $95^{\circ} \mathrm{C}$, followed by $10 \mathrm{~s}$ annealing at the indicated temperature, and $15 \mathrm{~s}$ amplification at $72^{\circ} \mathrm{C}$. Standard curves were determined by a serial dilution of a defined cDNA standard within each data set. Relative quantification was performed with the E-Method from Roche Applied Bioscience using the LightCycler ${ }^{\circledR} 480$ software 1.5 (Roche). 
2.4. Flow Cytometric Analysis. PBS supplemented with $0.2 \%$ BSA was used as assay buffer, and all steps of the staining process were performed at $4^{\circ} \mathrm{C}$. HUVECs were analyzed for expression of CX3CL1 by incubation with mouse monoclonal antibodies against human CX3CL1 $(5 \mu \mathrm{g} / \mathrm{ml})$ followed by incubation with APC-conjugated antimouse antibody $(1: 200)$. Isotype controls were used in parallel. The fluorescence signal was detected by flow cytometry (LRS Fortessa, BD Biosciences) and analyzed with FlowJo 8.7.3 software (Tree Star, Inc., Ashland, USA).

2.5. Apoptosis Assay. Endothelial cells were incubated with FITC-labelled annexin V in Hank's buffered salt solution (HBSS) for $30 \mathrm{~min}$. After washing with HBSS, at least 8 images of each well or $\mu$-Slide were made with the automated IncuCyte ZOOM microscope (Essen BioScience, Ann Arbor, USA). The relative apoptosis was determined by the ratio of the green fluorescence and the total cell confluence. The calculation of the green confluence and the total cell confluence was performed by the IncuCyte ZOOM microscope software 2014A (Essen BioScience).

2.6. ELISA Measurements. The release of human CX3CL1 and CXCL16 into the supernatant was analyzed by ELISA. Before the measurement, the culture supernatants were concentrated from $3 \mathrm{ml}$ to $0.5 \mathrm{ml}$ using Vivaspin 6 columns (10.000 MWCO) (Sartorius, Göttingen, Germany). 96 well plates were coated in PBS overnight at $4^{\circ} \mathrm{C}$ with a capture antibody against CX3CL1 $(4 \mu \mathrm{g} / \mathrm{ml})$ or CXCL16 $(1 \mu \mathrm{g} / \mathrm{ml})$, respectively. Before adding the samples, unspecific binding to the plate was blocked with washing buffer (PBS $+0.05 \%$ Tween) containing 2\% BSA for $2 \mathrm{~h}$ at room temperature. Samples were incubated for another $2 \mathrm{~h}$ at room temperature, and the bound chemokines were detected with a biotinylated secondary antibody against CX3CL1 $(0.3 \mu \mathrm{g} / \mathrm{ml})$ or CXCL16 $(0.5 \mu \mathrm{g} / \mathrm{ml})$. The chromogenic reaction was mediated by a standard procedure using $0.1 \mathrm{U} / \mathrm{ml}$ streptavidin-conjugated horseradish peroxidase (Roche, Basel, Switzerland) and the BM Blue POD substrate (Roche).

2.7. Flow Adhesion Assay. HUVECs were cultured for $24 \mathrm{~h}$ under flow conditions $\left(0.5\right.$ or $\left.30 \mathrm{dyn} / \mathrm{cm}^{2}\right)$ and stimulated with $10 \mathrm{ng} / \mathrm{ml} \mathrm{TNF} \alpha$ or left untreated for another $24 \mathrm{~h}$. In some experiments, cultivation was continued in the presence of an antibody against human CX3CL1 or an IgG control antibody $(10 \mu \mathrm{g} / \mathrm{ml})$ for 30 minutes at $37^{\circ} \mathrm{C}$. Subsequently, HUVECs were rinsed with flow adhesion buffer (10\% HBSS, $1 \%$ HEPES, and $1 \% \mathrm{BSA}$ in $\mathrm{H}_{2} \mathrm{O}$ ) for 3 minutes with a shear stress of $6 \mathrm{dyn} / \mathrm{cm}^{2}$. THP-1 cells were labelled for $30 \mathrm{~min}$ with $0.5 \mu \mathrm{M}$ Calcein AM, washed with PBS, and resuspended at a concentration of $0.5 \times 10^{6}$ cells in flow adhesion buffer. Labelled THP-1 cells were then perfused over the HUVECs with a flow rate of $1 \mathrm{ml} / \mathrm{min}$. Images of the $\mu$-Slides were taken during the perfusion, and adherent THP-1 cells were quantified using the IncuCyte ZOOM (Essen BioScience, Ann Arbor, USA). For each experiment, 8 images were taken and the average count per picture was used as result.

2.8. Statistics. Quantitative data are shown as mean $+\mathrm{SD}$ calculated from at least three independent experiments and for HUVECs and HUAECs also from different isolations. Data were analyzed by general mixed model analysis (PROC GLIMMIX, SAS 9.4, SAS Institute Inc., Cary, USA) and assumed to be derived from either log normal, negative binomial (count data), or beta (percentage data) distributions; residual plots and the Shapiro-Wilk test were used as diagnostics. In case of heteroscedasticity (according to the covtest statement), the degrees of freedom were adjusted by the Kenward-Roger approximation. The false discovery rate (FDR) was used to correct for multiple comparisons. Diagrams were created with PRISM 5.0 (GraphPad Software, La Jolla, USA). A $p$ value $<0.05$ was always considered significant.

\section{Results}

3.1. Endothelial CX3CL1 $m R N A$ and Protein Expression Is Downregulated under Flow Conditions. Human umbilical vein endothelial cells (HUVECs) were cultured under static or flow conditions. The resulting laminar shear stress at the endothelial surface was calculated from the different flow rates and flow chamber geometries, according to the specifications of the manufacturer. As expected, endothelial cells under flow conditions acquired an elongated shape and aligned in the direction of flow (Figure 1(a)). Analysis of CX3CL1 mRNA expression revealed that the chemokine is expressed to some degree under static culture conditions or conditions with low shear stress $\left(0.5 \mathrm{dyn} / \mathrm{cm}^{2}\right)$. This expression is downregulated when the cells were exposed to higher shear stress (Figure 1(b)). Consistent with this transcriptional regulation, released CX3CL1 protein was detected in the supernatant of static HUVEC culture, but considerably less CX3CL1 was found in supernatants from cultures exposed to higher shear stress (Figure 1(c)). These observations were made with HUVECs from at least five different donors.

To investigate whether the observed regulation of CX3CL1 would depend on the type and source of endothelial cells, we compared HUVECs with human umbilical artery endothelial cells (HUAECs) and human pulmonary microvascular endothelial cells (HPMECs). For all cell types, we observed constitutive expression of CX3CL1 mRNA when cells were cultured under static conditions. This expression was considerably decreased, when cells were exposed to high shear stress (Figure $1(\mathrm{~d})$ ).

We also studied the regulation of the other structurally related transmembrane chemokine CXCL16 in endothelial cells. This chemokine is also expressed at a constitutive level by endothelial cells, but the mRNA and protein expression is not affected by flow conditions. Further, there is no difference in HUVEC, HUAEC, or HPMECs (Figures 1(e)-1(g)).

These findings show that flow conditions downregulate the basal expression of CX3CL1 but not that of CXCL16 in endothelial cells from different vascular beds.

3.2. Inflammatory Induction of Endothelial CX3CL1 Is Suppressed under Flow Conditions. Stimulation with proinflammatory cytokines such as $\mathrm{TNF} \alpha$ has been reported to strongly upregulate the expression of CX3CL1 in endothelial cells [16]. We therefore questioned how flow conditions 


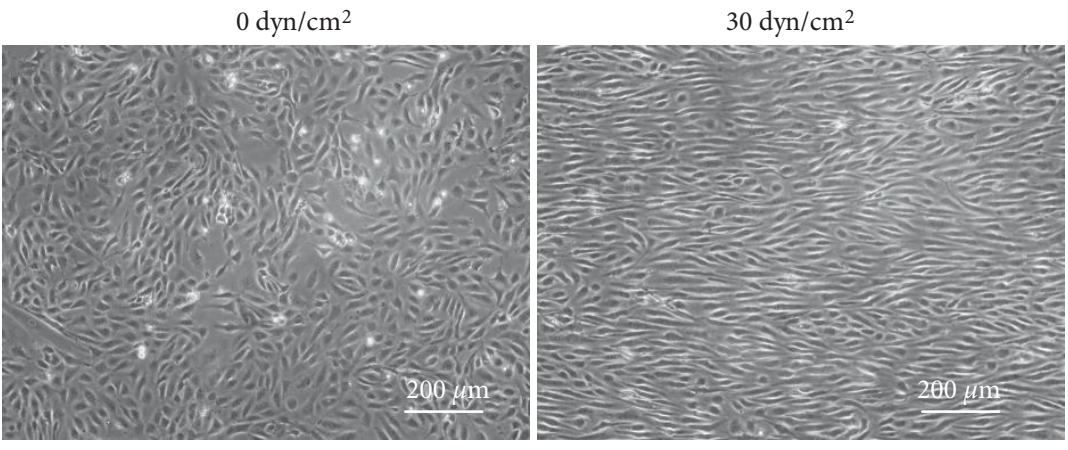

(a)

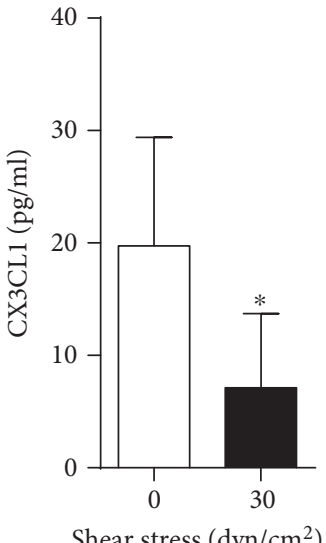

Shear stress $\left(\mathrm{dyn} / \mathrm{cm}^{2}\right)$

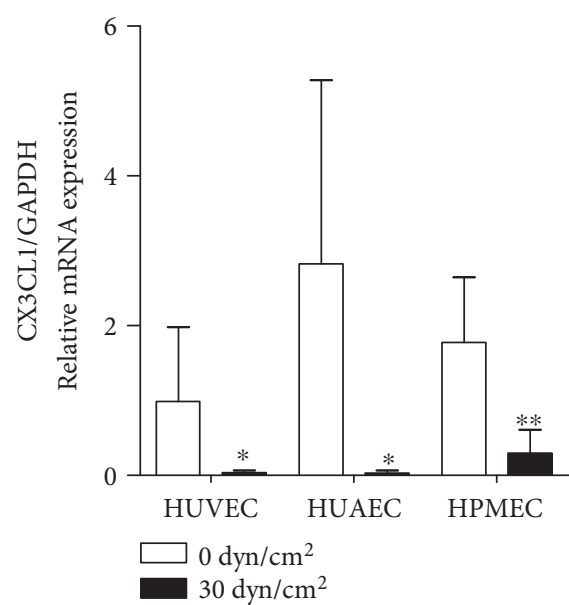

(d)

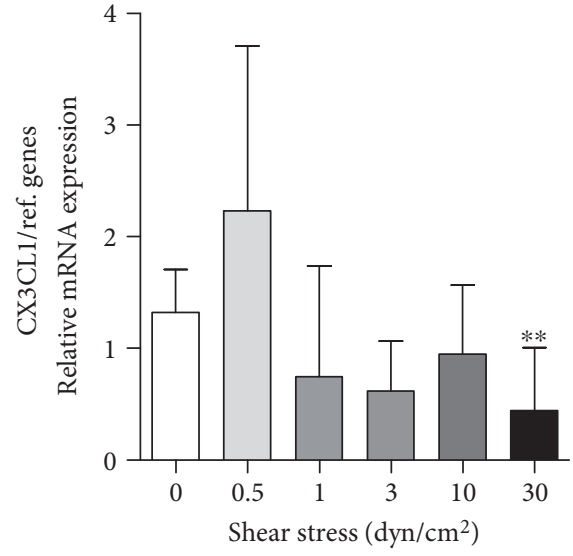

(b)

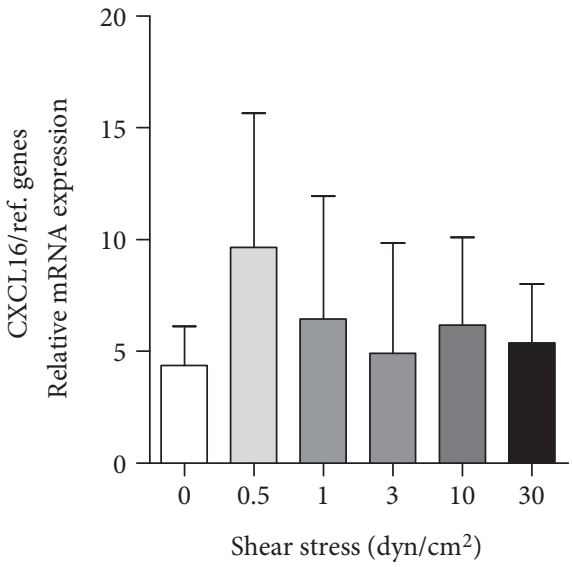

(e)

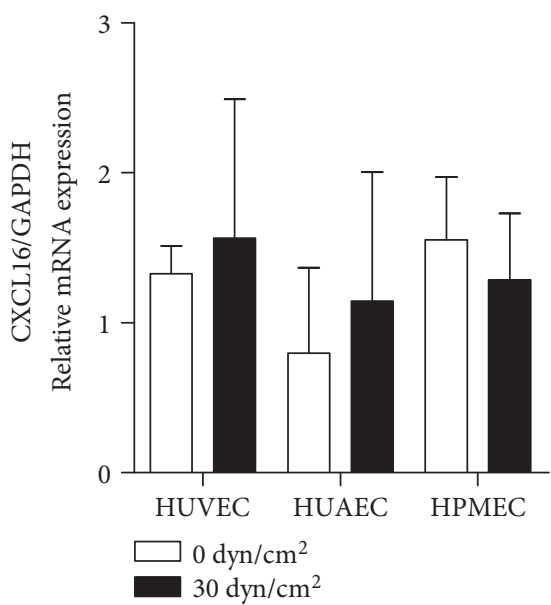

(f)

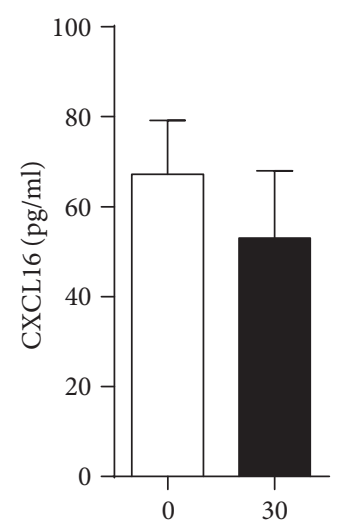

Shear stress $\left(\mathrm{dyn} / \mathrm{cm}^{2}\right)$

(g)

FIGURE 1: Regulation of constitutive CX3CL1 expression by shear stress. (a-c) HUVECs were cultured for $24 \mathrm{~h}$ under different conditions of flow resulting in the indicated laminar shear stress. Cells were then analyzed for their morphology by microscopy (a), for CX3CL1 mRNA expression (b), and their release of soluble CX3CL1 into the supernatant (c). (e, g) HUVECs were cultured under different flow conditions as described for $(\mathrm{a}-\mathrm{c})$ and analyzed for CXCL16 mRNA expression (e) and their release of soluble CXCL16 into the supernatant (g). (d, f) Endothelial cells from different vascular beds were cultured for $24 \mathrm{~h}$ with and without flow and analyzed for their CX3CL1 (d) or CXCL16 (f) mRNA expression. Data in (b-g) are shown as mean $+\mathrm{SD}$ for at least five different experiments. Statistical differences in comparison to the static control are indicated by asterisks $\left({ }^{*} p \leq 0.05,{ }^{* *} p \leq 0.01\right)$. 
would interfere with the upregulation of CX3CL1 expression in response to this cytokine. TNF $\alpha$ dose dependently induced CX3CL1 mRNA in endothelial cells (Figure 2(a)). A suboptimal dosage of $10 \mathrm{ng} / \mathrm{ml} \mathrm{TNF} \alpha$ was then chosen for flow experiments. As detected by annexin V staining, TNF $\alpha$ induced endothelial apoptosis was minimal under static conditions (less than $0.5 \%$ ) and almost absent under flow (Figure 2(b)).

Compared to static conditions, the upregulation of CX3CL1 by $\mathrm{TNF} \alpha$ was clearly suppressed at higher shear stress but not at low shear stress (Figure 2(c)). This suppression of mRNA expression correlated with a profound reduction of soluble CXC3L1 release into the supernatant and with a diminished surface expression of the transmembrane chemokine at conditions of higher shear stress (Figures 2(d) and 2(e)).

Notably, CXCL16 mRNA expression is only slightly upregulated by TNF $\alpha$ under static culture conditions. In contrast to CX3CL1, which is downregulated by flow conditions, CXCL16 seems to be slightly upregulated under flow conditions when cells are stimulated with TNF $\alpha$ (Figure 2(g)). However, this upregulation was not seen on the level of CXCL16 protein release (Figure 2(h)), which may be due to the high variability of CXCL16 release from the HUVEC preparations of the different donors and the fact that there is already a strong constitutive release of CXCL16, which might override the slight induction.

The data indicate that the exposure of endothelial cells to high shear stress can suppress the acquisition of a proinflammatory phenotype with respect to the induction of CX3CL1, but this does not account for CXCL16.

\subsection{Monocytic Cell Adhesion to Activated Endothelial Cells Is} Abrogated under Flow Conditions. Transmembrane CX3CL1 expressed on the endothelial surface can bind to its receptor CX3CR1 on monocytic cells and thereby mediate tight flow-resistant adhesion between both cell types. Cultured THP-1 cells express CX3CR1 and can be used as model for monocytic cell adhesion to endothelial cell-expressed CX3CL1 $[19,29]$. We sought to investigate how flow conditions would affect THP-1 cell adhesion and whether this is affected by the regulation of CX3CL1. HUVECs were grown and stimulated with or without TNF at flow conditions with low and high shear stress. Subsequently, THP-1 cells were perfused over the endothelial cells with the same flow rate for all conditions.

Only low adhesion was observed when endothelial cells were cultured at low shear stress and left unstimulated, but stimulation with $\mathrm{TNF} \alpha$ profoundly enhanced THP-1 cell adhesion (Figures 3(a) and 3(c)). This adhesion was at least in part mediated by CX3CL1 as evidenced by the incubation of the endothelial cells with a neutralizing antibody to CX3CL1 prior to adhesion experiments. This antibody-mediated inhibition reduced about one third of cell adhesion and was consistently observed in all experiments (Figure 3(b)).

When endothelial cells were grown and stimulated at conditions of high shear stress, THP-1 cell adhesion was almost completely abolished and no inhibitory effect of the neutralizing antibody could be observed (Figures 3(a) and 3(c)). These results demonstrate that culture under conditions of low pathologically relevant shear stress results in profound induction of cell adhesion upon proinflammatory stimulation. This adhesion is in part mediated by CX3CL1 and almost completely abolished when endothelial cells are exposed to higher but still physiological shear stress.

\section{Discussion}

Our present study demonstrates that the exposure of endothelial cells to conditions of high shear stress downregulates CX3CL1 mRNA and protein expression and prevents further upregulation of the chemokine in response to $\mathrm{TNF} \alpha$. By this, shear stress reduces the adhesiveness of endothelial cells for monocytes. Vice versa, static conditions or low shear stress allows constitutive expression of CX3CL1 in unstimulated cells and the upregulation of CX3CL1 in response to TNF $\alpha$, which then leads to increased monocytic cell recruitment.

Flow conditions have been shown to regulate a number of inflammatory mediators or adhesion molecules on the transcriptional level in endothelial cells. For example, the chemokine CCL-2/MCP-1 and the adhesion molecule VCAM-1 are downregulated under laminar flow conditions, even when cells are stimulated with $\mathrm{TNF} \alpha[11,12,30]$. We here show that constitutive and induced expression of the proinflammatory chemokine and adhesion molecule CX3CL1 is downregulated by shear stress. By contrast, we observed that the structurally and functionally related chemokine CXCL16 is not downregulated by flow conditions. This may be explained by the differences in the transcriptional regulation of both mediators. On the one hand, the transcription factor $\mathrm{NF}-\kappa \mathrm{B}$ seems to be involved in the induction of CX3CL1 by inflammatory stimuli [31]. On the other hand, regulatory activity of the transcription factor AP-1 has only been reported for CXCL16 [32, 33]. It is therefore likely that shear stress can prevent the induction of CX3CL1 by proinflammatory cytokines via an inhibitory mechanism on the involved transcription factors such as NF$\kappa \mathrm{B}$. A potential mediator would have been KLF2, which is directly induced by shear stress via MEF2 and the MAPK signaling pathway, and has been implicated in the induction of eNOS and in keeping endothelial cells in a noninflammatory state [34-36]. However, we observed no regulation of CX3CL1 in endothelial cells when KLF2 expression was induced with statins or inhibited with siRNA (unpublished observation). It is therefore questionable whether KLF2 negatively regulates CX3CL1, and it appears more likely that other transcriptional regulators suppress CX3CL1 expression under shear stress. Additionally, CX3CL1 can be regulated by TNF $\alpha$ on the level of RNA stability [37], and it is possible that this post-transcriptional regulation of CX3CL1 is affected by shear stress.

Monocyte recruitment is mediated by a number of effector molecules including adhesion molecules and chemokines. Of note, laminar shear stress reduces expression of CCL-2 which would result in reduced activation of adhesive mechanisms on monocytic cells such as upregulation of integrins $[38,39]$. Furthermore, integrin ligands such as VCAM-1 are downregulated on endothelial cells under flow conditions, which would further decrease cell adhesion [10-12]. In contrast to this mechanism, which requires the activation 


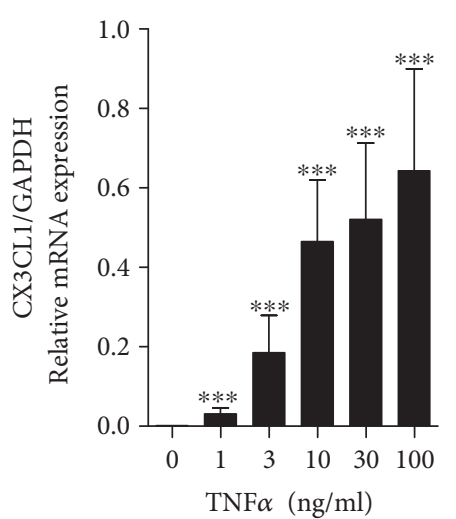

(a)

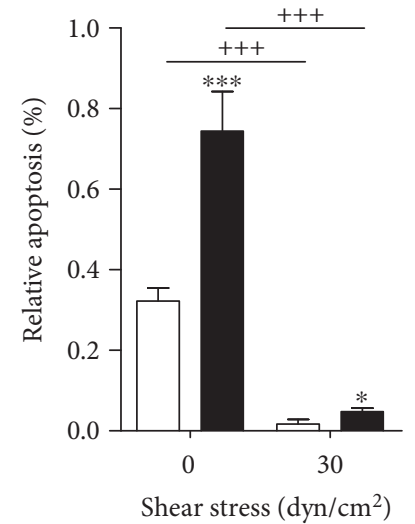

(b)

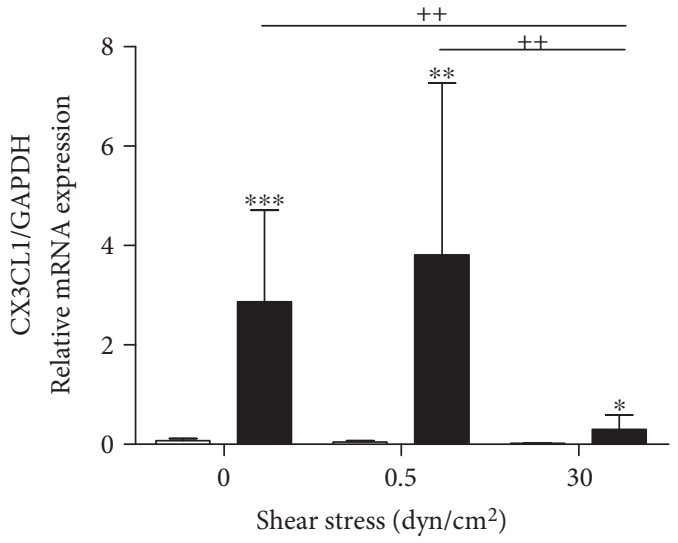

(c)
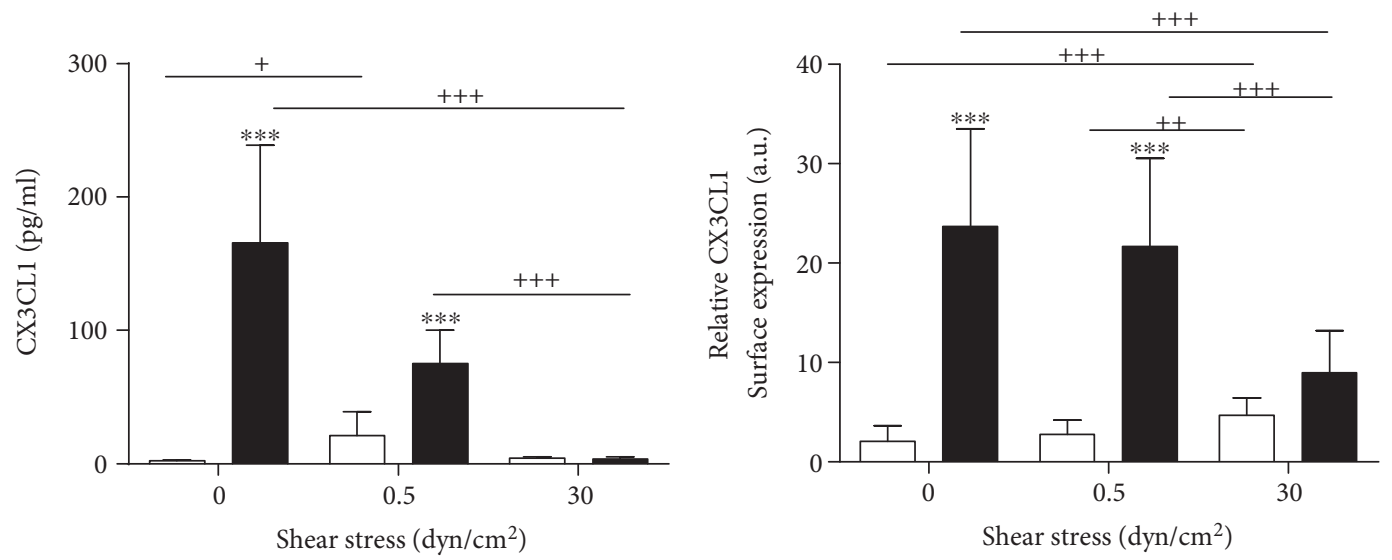

$\square$ Unstimulated

TNF $\alpha$

(d)

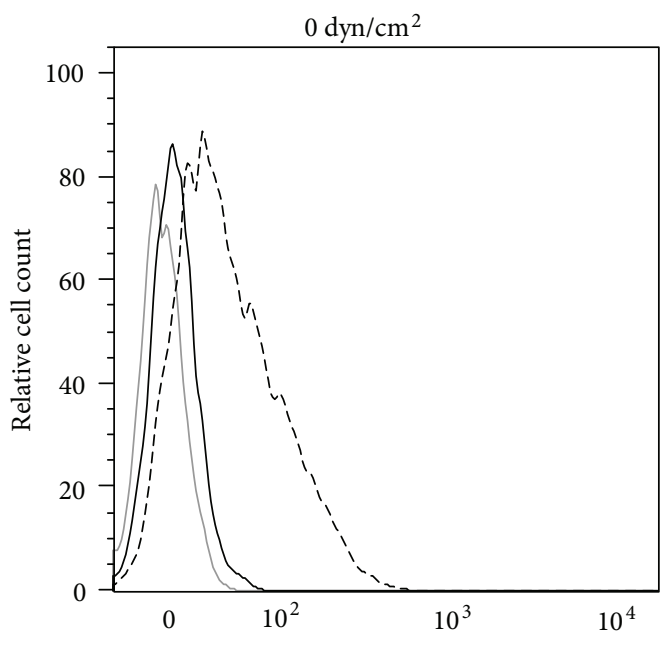

Fluorescence intensity

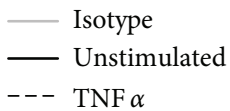

(e)

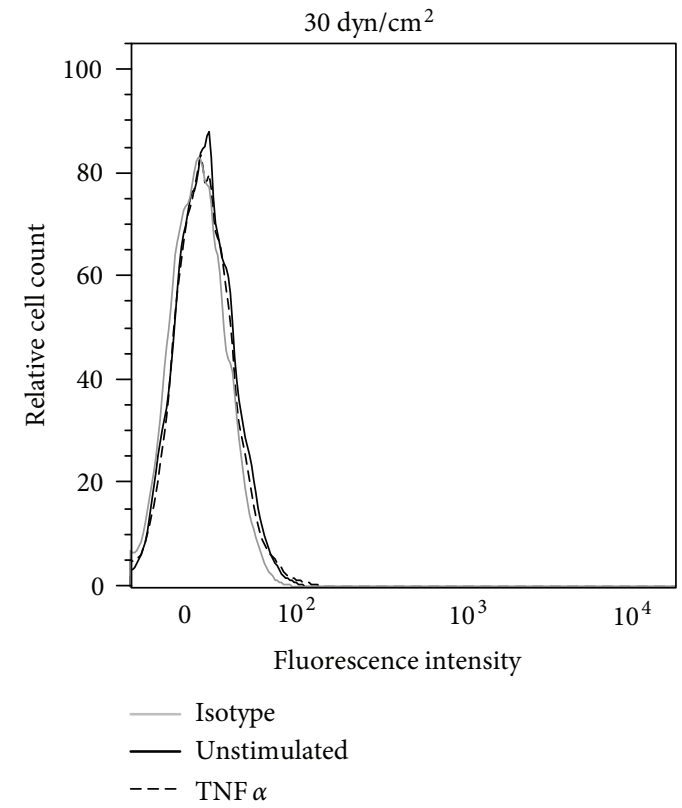

(f)

Figure 2: Continued. 


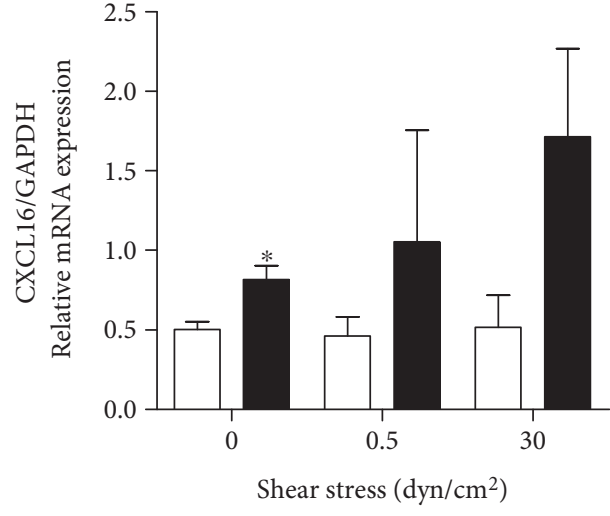

$(\mathrm{g})$

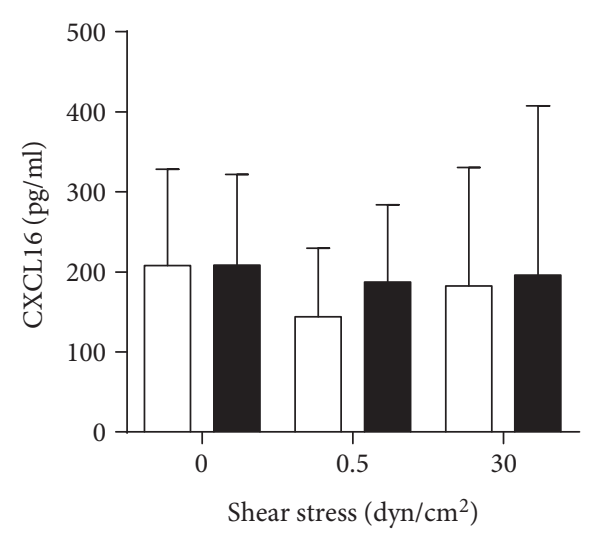

(h)

FIgURE 2: Regulation of induced CX3CL1 expression by shear stress. (a) HUVECs were stimulated with the indicated concentrations of TNF $\alpha$ for $24 \mathrm{~h}$ and analyzed for CX3CL1 mRNA expression. (b-f) HUVECs were cultured for $24 \mathrm{~h}$ and subsequently stimulated with or without $\mathrm{TNF} \alpha$ for $24 \mathrm{~h}$ at the indicated levels of shear stress. TNF $\alpha$-induced apoptosis was detected by annexin V staining (b). Cells were also analyzed for CX3CL1 mRNA expression (c) and release of CX3CL1 into the supernatant (d). CX3CL1 surface expression levels were determined by flow cytometry and are shown as summary of median fluorescence intensities and as representative histograms (e, f). (g, h) HUVECs were cultured and stimulated as described and analyzed for CXCL16 mRNA expression (g) and release of CXCL16 into the supernatant (h). Data are shown as mean + SD and are representative for at least three $(b, d, h)$ or four (a, c, e, g) different experiments. Statistical differences to the unstimulated control are indicated by asterisks $\left({ }^{*} p \leq 0.05,{ }^{* *} p \leq 0.01\right.$, and $\left.{ }^{* * *} p \leq 0.001\right)$ and differences between the flow conditions are indicated by crosses $\left({ }^{+} p \leq 0.05,{ }^{++} p \leq 0.01\right.$, and $\left.{ }^{+++} p \leq 0.001\right)$.

of monocytic cells, CX3CL1 can induce flow-resistant cell adhesion without the need of prior cell activation [18]. Additionally, CX3CL1 can activate monocytic cells to upregulate integrins and thereby tighten adhesive interactions and also induce transmigration through the endothelial cell layer $[19,40]$. By neutralizing surface-expressed CX3CL1 on endothelial cells, we could show that this CX3CL1-mediated mechanism is indeed relevant for monocyte adhesion to endothelial cells. However, we also observed CX3CL1-independent adhesion, which could be more dependent on integrin interaction with VCAM-1 on endothelial cells $[41,42]$. Importantly, both CX3CL1- and CX3CL1-independent adhesions were effectively suppressed when endothelial cells were exposed to shear stress. In addition to CX3CL1, CXCL16 can also promote cell adhesion via its receptor. The only known CXCL16 receptor CXCR6 is predominantly expressed on T cells but also on monocytic cells $[25,43]$. Yet the chemokine does not undergo regulation by shear stress as seen for CX3CL1. This may reflect that CXCL16 not only has proinflammatory functions but also acts as a scavenger receptor [44] with potentially protective functions in atherosclerotic lesions [24].

In healthy subjects, the expression of CX3CL1 is very low and soluble CX3CL1 is almost absent in the circulation [45]. This may be explained by the present findings showing that physiological flow conditions lower the constitutive expression and release of CX3CL1 by endothelial cells. Thus, to evaluate their physiological function in vitro, it is important to culture endothelial cells under flow conditions. Otherwise, the cells will acquire an inflammatory phenotype, which is more representative for pathophysiological situations.

Several studies have demonstrated that CX3CL1 is upregulated in subjects with vascular diseases [45, 46]. More soluble CX3CL1 can be found in the circulation, and this should at least in part be due to the increased expression and shedding of CX3CL1 by vascular cells. In fact, CX3CL1 is found on endothelial cells within sites of vascular inflammation. Notably, these sites often show disturbed blood flow where the shear stress may become less than $1 \mathrm{dyn} / \mathrm{cm}^{2}$. Here, endothelial cells show no proper alignment, more inflammatory phenotype, and more monocyte recruitment. In fact, within the carotid sinus of the brachiocephalic artery, the average shear stress is very low and this is a site of high CX3CL1 expression [27]. Experimental induction of low shear stress in carotid arteries in mice is associated with an upregulation of CX3CL1 expression [28]. Small molecules against CX3CR1 can prevent atherosclerosis in mice [47]. Moreover, knockout or inhibition of CX3CL1 in atherosclerosis-prone mice has demonstrated that the chemokine is critical especially for the recruitment of monocytes at these sites of reduced shear stress [27, 28]. These studies suggest that besides CX3CR1 antagonists, also targeting CX3CL1 on endothelial cells may represent a therapeutic option. The findings of our present study explain this site-specific function of CX3CL1 by providing in vitro evidence that the induction of CX3CL1 mRNA expression is profoundly suppressed by endothelial shear stress and only possible at sites where the shear stress is low or absent. Additionally, our data suggest that CX3CL1 should be implicated in very early states of atherogenesis where the disturbed flow enhances the susceptibility of the still-intact endothelium to respond to inflammatory triggers by the upregulation of CX3CL1. Nevertheless, low shear stress is not sufficient to initiate the inflammatory process since effective CX3CL1 induction requires the presence of inflammatory stimuli. This finding is consistent with the concept that low shear stress is only one of many proinflammatory factors which need to synergize for shifting the balance from atheroprotection towards atherogenesis by upregulating various effector molecules including CX3CL1. 


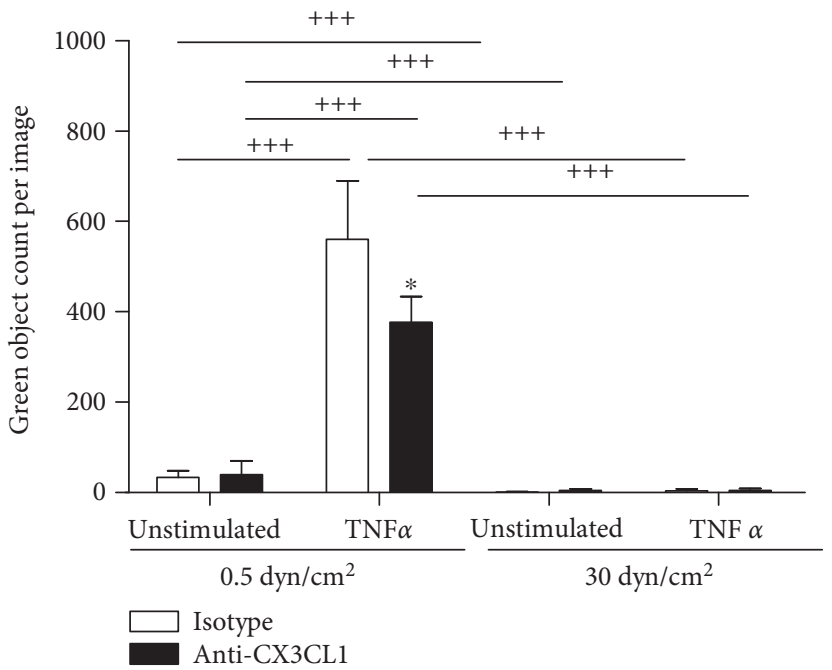

(a)

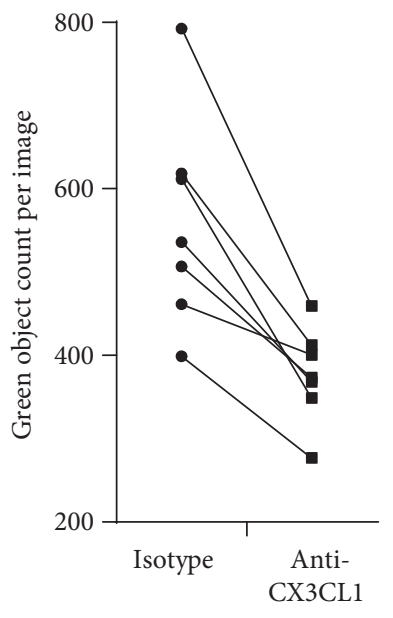

(b)

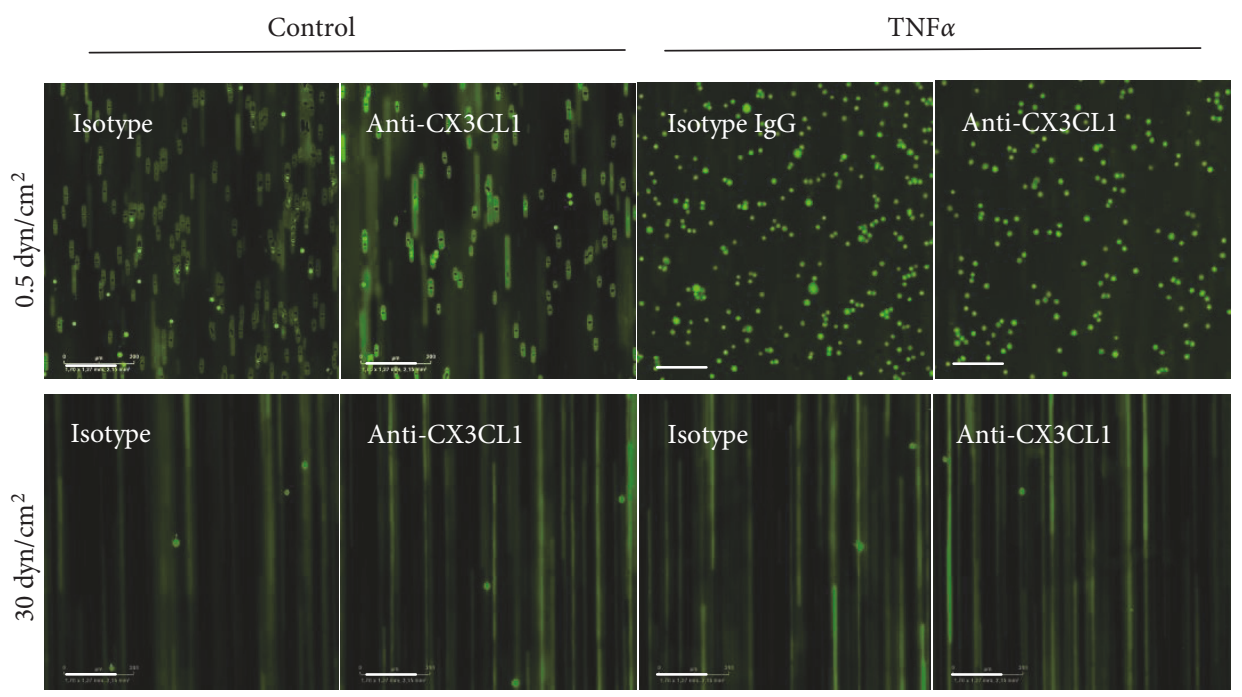

Scale $=100 \mu \mathrm{m}$

(c)

FIGURE 3: Regulation of CX3CL1 dependent THP-1 cell adhesion by shear stress. (a-c) HUVECs were cultured for $24 \mathrm{~h}$ and subsequently stimulated with or without TNF $\alpha$ for $24 \mathrm{~h}$ at the indicated levels of shear stress. Cells were then treated with a neutralizing antibody against CX3CL1 or an IgG isotype control for $0.5 \mathrm{~h}$. Subsequently, fluorescently labelled THP-1 cells were perfused over the endothelial cell layer. THP-1 cells were visualized by fluorescence microscopy, and for each experiment adhered, THP-1 cells were counted in at least 8 images (a). Data are shown as mean + SD of 3-8 experiments as indicated. Statistical differences in comparison to the IgG isotype control or the specified flow conditions are indicated by asterisks $\left({ }^{*} p \leq 0.05\right)$ or crosses $\left({ }^{+++} p \leq 0.001\right)$, respectively. (b) The effect of the inhibitory antibody against CX3CL1 is shown for each experiment performed with a different endothelial isolate. (c) Representative images showing adherent THP-1 cells (green bright circular objects) and flowing THP-1 cells (green lines) for all conditions.

\section{Conclusion}

In summary, our study demonstrates that high laminar shear stress downregulates endothelial CX3CL1 mRNA and protein expression and even attenuates its induction mediated by the inflammatory cytokine TNF $\alpha$. As a result, laminar shear stress reduces the adhesiveness of endothelial cells for CX3CR1positive monocytes. By this mechanism, shear stress can counteract vascular inflammation.

\section{Conflicts of Interest}

The authors declare that they have no conflicts of interest.

\section{Acknowledgments}

The authors thank Tanja Woopen and Melanie Esser for expert technical assistance. This work was supported by the IZKF (project IZKF-T11-5 of Andreas Ludwig and Daniela 
Dreymueller), the ERS boost fund (project OPBF071 of Andreas Ludwig and Daniela Dreymueller) of the RWTH Aachen, and the Deutsche Forschungsgemeinschaft (project LU869/7-1 of Andreas Ludwig).

\section{References}

[1] T. G. Papaioannou and C. Stefanadis, "Vascular wall shear stress: basic principles and methods," Hellenic Journal of Cardiology, vol. 46, no. 1, pp. 9-15, 2005.

[2] A. G. Koutsiaris, S. V. Tachmitzi, and N. Batis, "Wall shear stress quantification in the human conjunctival pre-capillary arterioles in vivo," Microvascular Research, vol. 85, pp. 34-39, 2013.

[3] K. A. Barbee, P. F. Davies, and R. Lal, "Shear stress-induced reorganization of the surface topography of living endothelial cells imaged by atomic force microscopy," Circulation Research, vol. 74, no. 1, pp. 163-171, 1994.

[4] S. Dimmeler, B. Assmus, C. Hermann, J. Haendeler, and A. M. Zeiher, "Fluid shear stress stimulates phosphorylation of Akt in human endothelial cells: involvement in suppression of apoptosis," Circulation Research, vol. 83, no. 3, pp. 334-341, 1998.

[5] S. Akimoto, M. Mitsumata, T. Sasaguri, and Y. Yoshida, "Laminar shear stress inhibits vascular endothelial cell proliferation by inducing cyclin-dependent kinase inhibitor p21(Sdi1/Cip1/ Waf1)," Circulation Research, vol. 86, no. 2, pp. 185-190, 2000.

[6] B. S. Conklin, R. P. Vito, and C. Chen, "Effect of low shear stress on permeability and occludin expression in porcine artery endothelial cells," World Journal of Surgery, vol. 31, no. 4, pp. 733-743, 2007.

[7] N. G. dela Paz, T. E. Walshe, L. L. Leach, M. Saint-Geniez, and P. A. D'Amore, "Role of shear-stress-induced VEGF expression in endothelial cell survival," Journal of Cell Science, vol. 125, no. Pt 4, pp. 831-843, 2012.

[8] M. E. Davis, H. Cai, G. R. Drummond, and D. G. Harrison, "Shear stress regulates endothelial nitric oxide synthase expression through c-Src by divergent signaling pathways," Circulation Research, vol. 89, no. 11, pp. 1073-1080, 2001.

[9] R. J. Dekker, J. V. van Thienen, J. Rohlena et al., "Endothelial KLF2 links local arterial shear stress levels to the expression of vascular tone-regulating genes," The American Journal of Pathology, vol. 167, no. 2, pp. 609-618, 2005.

[10] A. Ohtsuka, J. Ando, R. Korenaga, A. Kamiya, N. ToyamaSorimachi, and M. Miyasaka, "The effect of flow on the expression of vascular adhesion molecule-1 by cultured mouse endothelial cells," Biochemical and Biophysical Research Communications, vol. 193, no. 1, pp. 303-310, 1993.

[11] P. S. Tsao, R. Buitrago, J. R. Chan, and J. P. Cooke, "Fluid flow inhibits endothelial adhesiveness. Nitric oxide and transcriptional regulation of VCAM-1," Circulation, vol. 94, no. 7, pp. 1682-1689, 1996.

[12] J. S. DeVerse, A. S. Sandhu, N. Mendoza et al., "Shear stress modulates VCAM-1 expression in response to TNF- $\alpha$ and dietary lipids via interferon regulatory factor-1 in cultured endothelium," American Journal of Physiology. Heart and Circulatory Physiology, vol. 305, no. 8, pp. H1149-H1157, 2013.

[13] G. Dai, M. R. Kaazempur-Mofrad, S. Natarajan et al., "Distinct endothelial phenotypes evoked by arterial waveforms derived from atherosclerosis-susceptible and -resistant regions of human vasculature," Proceedings of the National Academy of Sciences of the United States of America, vol. 101, no. 41, pp. 14871-14876, 2004.
[14] P. Libby, "Inflammation in atherosclerosis," Nature, vol. 420, no. 6917, pp. 868-874, 2002.

[15] A. Ludwig and C. Weber, "Transmembrane chemokines: versatile "special agents" in vascular inflammation," Thrombosis and Haemostasis, vol. 98, no. 5, pp. 262-273, 2007.

[16] J. F. Bazan, K. B. Bacon, G. Hardiman et al., "A new class of membrane-bound chemokine with a CX3C motif," Nature, vol. 385, no. 6617, pp. 640-644, 1997a.

[17] T. Imai, K. Hieshima, C. Haskell et al., "Identification and molecular characterization of fractalkine receptor CX3CR1, which mediates both leukocyte migration and adhesion," Cell, vol. 91, no. 4, pp. 521-530, 1997.

[18] A. M. Fong, L. A. Robinson, D. A. Steeber et al., "Fractalkine and CX3CR1 mediate a novel mechanism of leukocyte capture, firm adhesion, and activation under physiologic flow," The Journal of Experimental Medicine, vol. 188, no. 8, pp. 1413-1419, 1998.

[19] N. Schwarz, J. Pruessmeyer, F. M. Hess et al., "Requirements for leukocyte transmigration via the transmembrane chemokine CX3CL1," Cellular and Molecular Life Sciences, vol. 67, no. 24, pp. 4233-4248, 2010.

[20] C. Hundhausen, D. Misztela, T. A. Berkhout et al., "The disintegrin-like metalloproteinase ADAM10 is involved in constitutive cleavage of CX3CL1 (fractalkine) and regulates CX3CL1-mediated cell-cell adhesion," Blood, vol. 102, no. 4, pp. 1186-1195, 2003.

[21] K. J. Garton, P. J. Gough, C. P. Blobel et al., "Tumor necrosis factor-alpha-converting enzyme (ADAM17) mediates the cleavage and shedding of fractalkine (CX3CL1)," The Journal of Biological Chemistry, vol. 276, no. 41, pp. 37993-38001, 2001.

[22] O. Hofnagel, T. Engel, N. J. Severs, H. Robenek, and I. Buers, "SR-PSOX at sites predisposed to atherosclerotic lesion formation mediates monocyte-endothelial cell adhesion," Atherosclerosis, vol. 217, no. 3, pp. 371-378, 2011.

[23] S. Abel, C. Hundhausen, R. Mentlein et al., "The transmembrane CXC-chemokine ligand 16 is induced by IFN-gamma and TNF-alpha and shed by the activity of the disintegrinlike metalloproteinase ADAM10," Journal of Immunology, vol. 172, no. 10, pp. 6362-6372, 2004.

[24] A. M. Aslanian and I. F. Charo, "Targeted disruption of the scavenger receptor and chemokine CXCL16 accelerates atherosclerosis," Circulation, vol. 114, no. 6, pp. 583-590, 2006.

[25] E. Galkina, B. L. Harry, A. Ludwig et al., "CXCR6 promotes atherosclerosis by supporting T-cell homing, interferongamma production, and macrophage accumulation in the aortic wall," Circulation, vol. 116, no. 16, pp. 1801-1811, 2007.

[26] P. Lesnik, C. A. Haskell, and I. F. Charo, "Decreased atherosclerosis in CX3CR1-/- mice reveals a role for fractalkine in atherogenesis," The Journal of Clinical Investigation, vol. 111, no. 3, pp. 333-340, 2003.

[27] D. Teupser, S. Pavlides, M. Tan, J.-C. Gutierrez-Ramos, R. Kolbeck, and J. L. Breslow, "Major reduction of atherosclerosis in fractalkine (CX3CL1)-deficient mice is at the brachiocephalic artery, not the aortic root," Proceedings of the National Academy of Sciences of the United States of America, vol. 101, no. 51, pp. 17795-17800, 2004.

[28] C. Cheng, D. Tempel, R. Van Haperen et al., "Shear stress induced changes in atherosclerotic plaque composition are modulated by chemokines," The Journal of Clinical Investigation, vol. 117, no. 3, pp. 616-626, 2007. 
[29] G. A. Chapman, K. E. Moores, J. Gohil et al., "The role of fractalkine in the recruitment of monocytes to the endothelium," European Journal of Pharmacology, vol. 392, no. 3, pp. 189195, 2000.

[30] K. Urschel, I. Cicha, W. G. Daniel, and C. D. Garlichs, "Shear stress patterns affect the secreted chemokine profile in endothelial cells," Clinical Hemorheology and Microcirculation, vol. 50, no. 1-2, pp. 143-152, 2012.

[31] G. E. Garcia, Y. Xia, S. Chen et al., "NF-kappaB-dependent fractalkine induction in rat aortic endothelial cells stimulated by IL-1beta, TNF-alpha, and LPS," Journal of Leukocyte Biology, vol. 67, no. 4, pp. 577-584, 2000.

[32] S. H. Ko, J. I. Jeon, H. Kim, Y. J. Kim, J. Youn, and J. M. Kim,

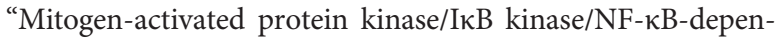
dent and AP-1-independent CX3CL1 expression in intestinal epithelial cells stimulated with Clostridium difficile toxin A," Journal of Molecular Medicine, vol. 92, no. 4, pp. 411-427, 2014.

[33] B. Chandrasekar, S. Bysani, and S. Mummidi, "CXCL16 signals via Gi, phosphatidylinositol 3-kinase, Akt, I kappa B kinase, and nuclear factor-kappa B and induces cell-cell adhesion and aortic smooth muscle cell proliferation," The Journal of Biological Chemistry, vol. 279, no. 5, pp. 3188-3196, 2004.

[34] R. J. Dekker, S. Van Soest, R. D. Fontijn et al., "Prolonged fluid shear stress induces a distinct set of endothelial cell genes, most specifically lung Krüppel-like factor (KLF2)," Blood, vol. 100, no. 5, pp. 1689-1698, 2002.

[35] K. M. Parmar, H. B. Larman, G. Dai et al., "Integration of flow-dependent endothelial phenotypes by Kruppel-like factor 2," The Journal of Clinical Investigation, vol. 116, no. 1, pp. 49-58, 2006.

[36] J. O. Fledderus, R. A. Boon, O. L. Volger et al., "KLF2 primes the antioxidant transcription factor Nrf2 for activation in endothelial cells," Arteriosclerosis, Thrombosis, and Vascular Biology, vol. 28, no. 7, pp. 1339-1346, 2008.

[37] T. Matsumiya, K. Ota, T. Imaizumi, H. Yoshida, H. Kimura, and K. Satoh, "Characterization of synergistic induction of CX3CL1/ fractalkine by TNF-alpha and IFN-gamma in vascular endothelial cells: an essential role for TNF-alpha in post-transcriptional regulation of CX3CL1," Journal of Immunology, vol. 184, no. 8, pp. 4205-4214, 2010.

[38] N. Ashida, H. Arai, M. Yamasaki, and T. Kita, "Distinct signaling pathways for MCP-1-dependent integrin activation and chemotaxis," The Journal of Biological Chemistry, vol. 276, no. 19, pp. 16555-16560, 2001.

[39] J. O. Fledderus, J. V. Van Thienen, R. A. Boon et al., "Prolonged shear stress and KLF2 suppress constitutive proinflammatory transcription through inhibition of ATF2," Blood, vol. 109, no. 10, pp. 4249-4257, 2007.

[40] S. Goda, T. Imai, O. Yoshie et al., "CX3C-chemokine, fractalkine-enhanced adhesion of THP-1 cells to endothelial cells through integrin-dependent and -independent mechanisms," Journal of Immunology, vol. 164, no. 8, pp. 43134320, 2000.

[41] M. J. Elices, L. Osborn, Y. Takada et al., "VCAM-1 on activated endothelium interacts with the leukocyte integrin VLA-4 at a site distinct from the VLA-4/fibronectin binding site," Cell, vol. 60, no. 4, pp. 577-584, 1990.

[42] R. Alon, P. D. Kassner, M. W. Carr, E. B. Finger, M. E. Hemler, and T. A. Springer, "The integrin VLA-4 supports tethering and rolling in flow on VCAM-1," The Journal of Cell Biology, vol. 128, no. 6, pp. 1243-1253, 1995.
[43] A. Wilbanks, S. C. Zondlo, K. Murphy et al., "Expression cloning of the STRL33/BONZO/TYMSTR ligand reveals elements of CC, CXC, and CX3C chemokines," Journal of Immunology, vol. 166, no. 8, pp. 5145-5154, 2001.

[44] T. Shimaoka, N. Kume, M. Minami et al., "Molecular cloning of a novel scavenger receptor for oxidized low density lipoprotein, SR-PSOX, on macrophages," The Journal of Biological Chemistry, vol. 275, no. 52, pp. 40663-40666, 2000.

[45] S. Matsumura, B. Wang, N. Kawashima et al., "Radiationinduced CXCL16 release by breast cancer cells attracts effector T cells," Journal of Immunology, vol. 181, no. 5, pp. 30993107, 2008.

[46] M. Stolla, J. Pelisek, M. L. von Brühl et al., "Fractalkine is expressed in early and advanced atherosclerotic lesions and supports monocyte recruitment via CX3CR1," PloS One, vol. 7, no. 8, pp. 1-9, 2012.

[47] L. Poupel, A. Boissonnas, P. Hermand et al., "Pharmacological inhibition of the chemokine receptor, CX3CR1, reduces atherosclerosis in mice," Arteriosclerosis, Thrombosis, and Vascular Biology, vol. 33, no. 10, pp. 2297-2305, 2013. 


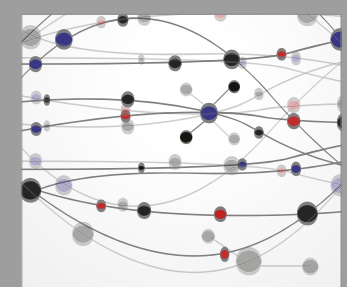

The Scientific World Journal
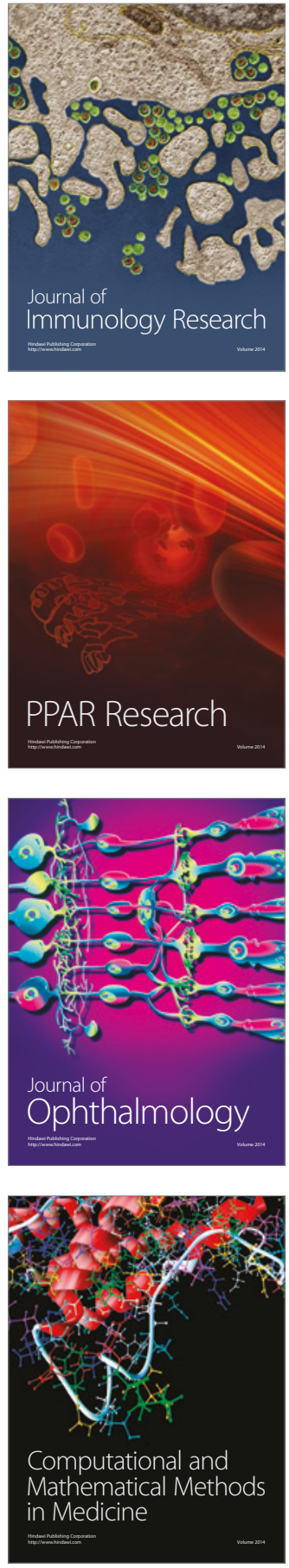

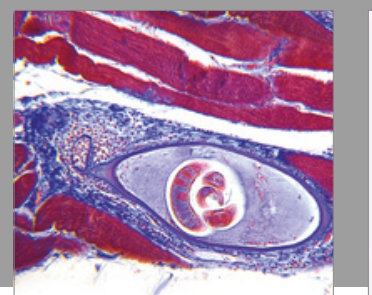

Gastroenterology Research and Practice
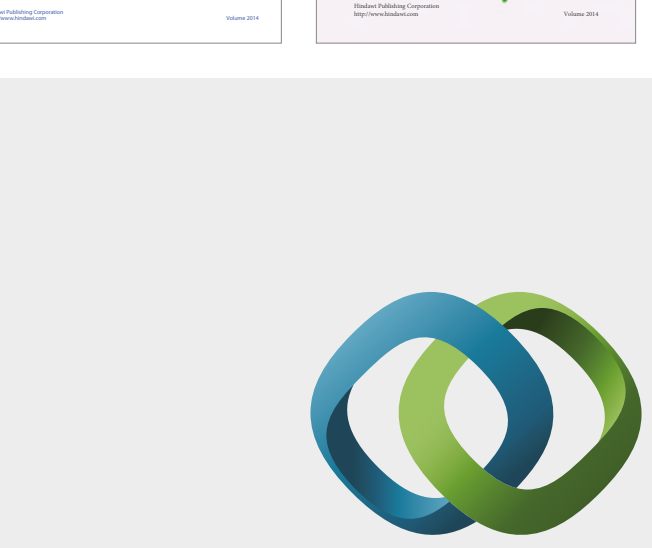

\section{Hindawi}

Submit your manuscripts at

https://www.hindawi.com
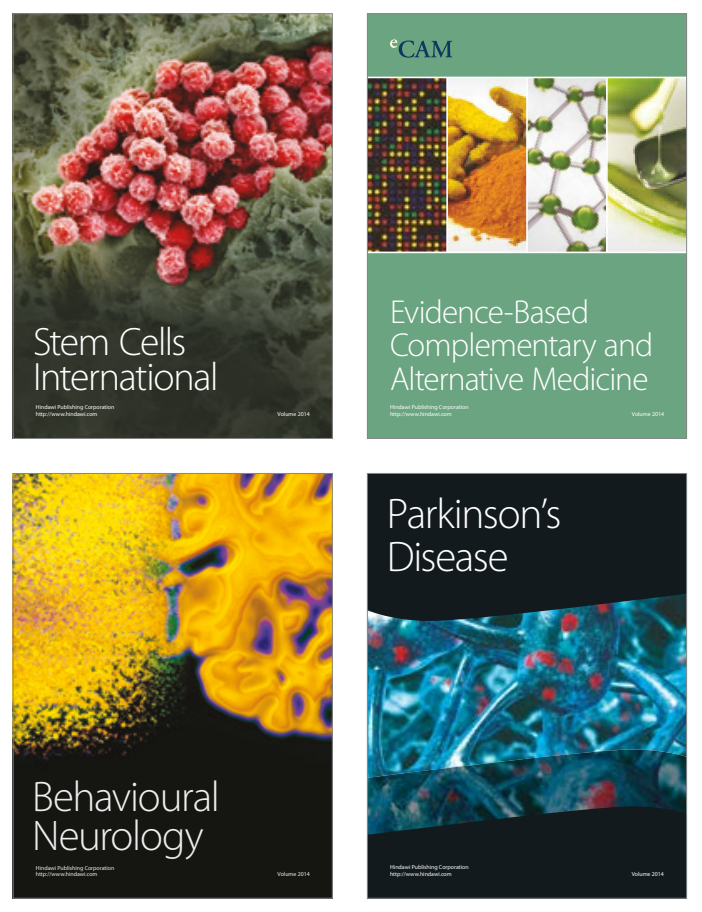
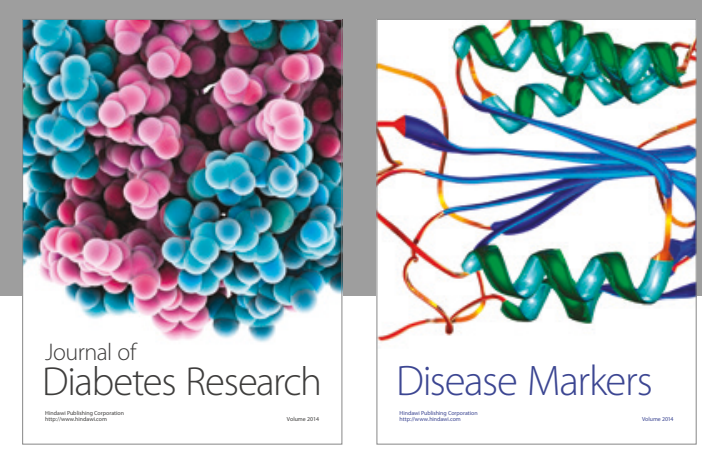

Disease Markers
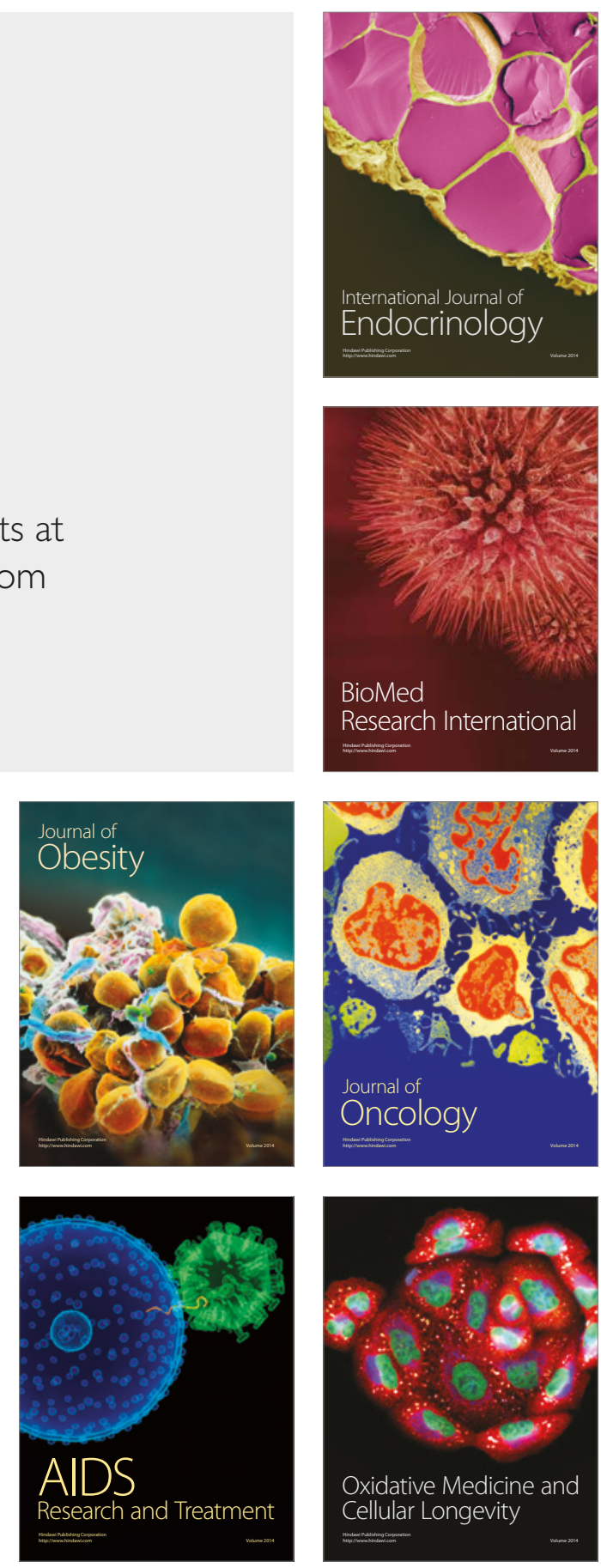